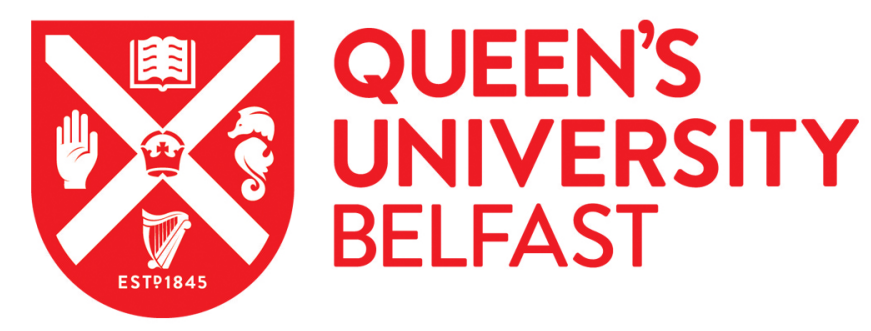

\title{
Calculation of gamma spectra for positron annihilation on molecules
}

Green, D. G., Saha, S., Wang, F., Gribakin, G. F., \& Surko, C. M. (2011). Calculation of gamma spectra for positron annihilation on molecules. In Materials Science Forum (Vol. 666, pp. 21-24) https://doi.org/10.4028/www.scientific.net/MSF.666.21

\section{Published in:}

Materials Science Forum

\section{Document Version:}

Peer reviewed version

Queen's University Belfast - Research Portal:

Link to publication record in Queen's University Belfast Research Portal

\footnotetext{
General rights

Copyright for the publications made accessible via the Queen's University Belfast Research Portal is retained by the author(s) and / or other copyright owners and it is a condition of accessing these publications that users recognise and abide by the legal requirements associated with these rights.

Take down policy

The Research Portal is Queen's institutional repository that provides access to Queen's research output. Every effort has been made to ensure that content in the Research Portal does not infringe any person's rights, or applicable UK laws. If you discover content in the Research Portal that you believe breaches copyright or violates any law, please contact openaccess@qub.ac.uk.
} 


\title{
Calculation of gamma spectra for positron annihilation on molecules
}

\author{
D.G. Green ${ }^{1, a}$, S. Saha², F. Wang ${ }^{2}$, G.F Gribakin ${ }^{1}$, C.M. Surko ${ }^{3}$ \\ ${ }^{1}$ Department of Applied Mathematics and Theoretical Physics, Queen's University Belfast, Belfast, \\ BT71NN, Northern Ireland, UK \\ ${ }^{2}$ Swinburne University of Technology, Melbourne, Victoria 3122, Australia \\ ${ }^{3}$ Physics Department, University of California, San Diego, La Jolla, California 92093-0319, USA \\ adgreen09@qub.ac.uk
}

Keywords: Positron annihilation, molecules, gamma spectra, electron momentum density

\begin{abstract}
Calculations of gamma spectra for positron annihilation for a selection of molecules, including methane and its fluoro-substitutes, ethane, propane, butane and benzene are presented. The contribution to the $\gamma$-spectra from individual molecular orbitals is obtained from electron momentum distributions calculated using the density functional theory (DFT) based B3LYP/TZVP model. For positrons thermalised to room temperature, the calculation, in its simplest form, effectively treats the positron as a plane wave and gives positron annihilation $\gamma$-spectra linewidths that are broader (30-40\%) than experiment, although the main chemical trends are reproduced. The main physical reason for this is the neglect of positron repulsion from the nuclei. We show that this effect can be incorporated through momentum-dependent correction factors, determined from positron-atom calculations, e.g., many-body perturbation theory. Inclusion of these factors in the calculation gives linewidths that are in improved agreement with experiment.
\end{abstract}

\section{Introduction}

Despite an exhaustive set of experimental results [1], there have been very few theoretical calculations of the $\gamma$-spectra for positron annihilation on molecules [2]. This situation is in sharp contrast with positron annihilation studies in solids, where much understanding has been achieved over the past 50 years $[3,4]$. In this work we use modern quantum-chemistry methods to calculate positron annihilation spectra for molecules, and analyse the results using simpler approaches, such as LCAO (linear combination of atomic orbitals).

In the dominant annihilation process, a positron annihilates with an atomic or molecular electron in an orbital $n$ to produce two gamma photons with total momentum P. Non-zero values of P lead to Doppler shifts of the photon energy from the electron rest energy of $m c^{2}=511 \mathrm{keV}: E_{\gamma}=m c^{2}+$ $m c V \cos \theta$, where $\mathbf{V}=\mathrm{P} / 2 m$ is the centre of mass velocity of the electron-positron pair and $\theta$ is the angle between the direction of the photon and $\mathbf{V}$. Assuming that $V \ll c$, and $p_{\gamma}=E_{\gamma} / c \approx m c$, the shift of the photon energy from the centre of the line is $\varepsilon \equiv E_{\gamma}-m c^{2}=1 / 2 P c \cos \theta$.

For low energy positrons, the effect of Doppler broadening is mostly due to the electron momenta. It can be used to characterize the electron orbitals involved in the annihilation [1]. However, the positron perturbs the system with the consequence that the $\gamma$-spectra are also sensitive to its presence as well as to additional correlation effects. 


\section{Theory}

The photon Doppler shift spectrum is

$$
w(\varepsilon)=\frac{1}{c} \int_{2|\varepsilon| / c}^{\infty} \rho_{n}^{a}(P) P d P,
$$

where $\rho_{n}^{a}(P)$ is the annihilation momentum density,

$$
\rho_{n}^{a}(P)=\int\left|A_{n}(\mathbf{P})\right|^{2} \frac{d \Omega_{\mathbf{P}}}{(2 \pi)^{3}},
$$

and $A_{n}(\mathrm{P})$ is the annihilation amplitude, e.g., $A_{n}(\mathrm{P})=\int d \mathbf{r} e^{-i \mathbf{P} . \mathbf{r}} \psi_{n}(\mathbf{r}) \varphi_{\mathbf{k}}(\mathbf{r})$, in the independent particle approximation, $\psi_{n}$ and $\varphi_{\mathbf{k}}$ being the electron and positron wavefunctions, respectively. In the planewave (PW) approximation, $\varphi_{\mathbf{k}}=e^{\mathbf{i k} \cdot \mathbf{r}}$, and for positrons thermalized to room temperature (with typical momenta $k \simeq 0.04$ a.u., $\left.\varphi_{\mathbf{k}} \simeq 1\right), \rho_{n}^{a}(P)$ reduces to the electron momentum density of orbital $n, \rho_{n}(P)$, such as that measured in electron momentum spectroscopy [5]. Using the DFT based B3LYP/TZVP model (Becke 3-parameter Lee-Yang-Parr exchange-correlation functional, polarized valence triple- $\zeta$ basis) to calculate the electron momentum densities we find that the calculated spectra are approximately $30-40 \%$ broader than experiment (see Fig. 1). As shown below, the main reason for this discrepancy is the neglect of the positron repulsion from the nuclei. It leads to an overestimate of the contribution of small positron-nuclear separations where the electron momentum is large.

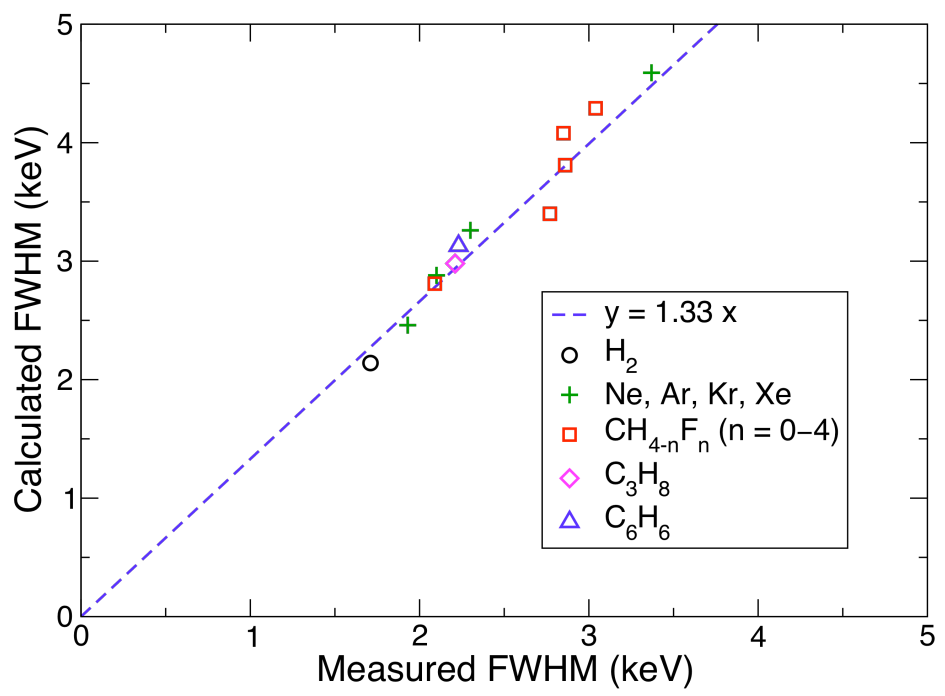

Figure 1: Full width at half maximum (FWHM) of the positron annihilation spectra calculated in the independent-particle approximation with $\varphi_{\mathrm{k}} \simeq 1$ vs. measured values [1]. Dashed line shows that the calculated values in this approximation are about $33 \%$ greater than experimental.

To investigate the effect of the nuclear repulsion and additional correlation effects we employ a linear combination of atomic orbital description (LCAO) for the molecular orbitals:

$$
\psi_{n}(\mathbf{r})=\sum_{i} c_{i} \phi_{i}\left(\mathbf{r}-\mathbf{R}_{i}\right),
$$

where $c_{i}$ are the expansion coefficients and $\phi_{i}\left(\mathbf{r}-\mathbf{R}_{i}\right)$ are atomic orbitals centred on $\mathbf{R}_{i}$. Substituting Eqn. (3) into Eqn. (2) under the assumptions of a unit positron wavefunction allows the molecular annihilation momentum density to be written in terms of atomic momentum densities (AMD) [6]. The AMD of a particular atomic orbital can then be corrected to account for the effects of the true positron wavefunction by a momentum-dependent atomic adjustment factor $G_{i}(P)$. These factors 


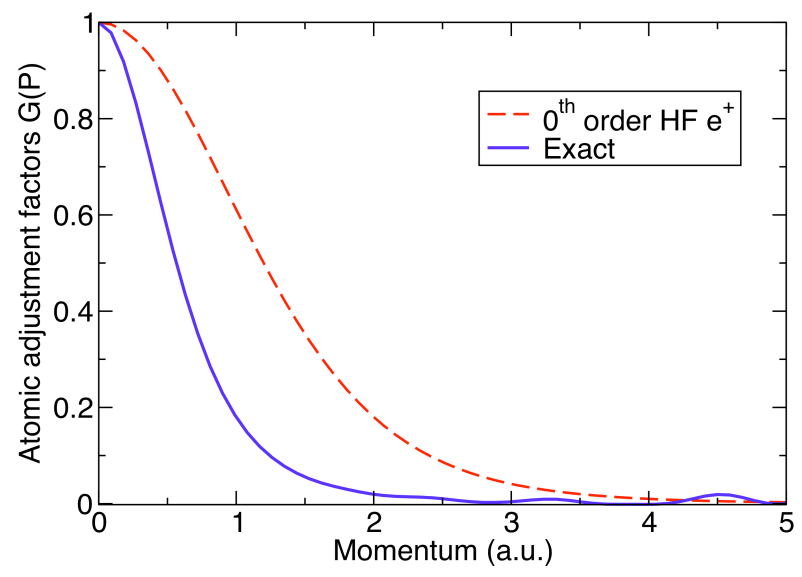

Figure 2: Momentum-dependent adjustment factor of atomic hydrogen calculated in the HF approximation (dashed line) and by MBPT ("exact", solid line) using the method of $[7,8]$.

are defined as the ratio of the annihilation momentum density for an atomic orbital $i$ (in which the positron wavefunction is accounted for $), \rho_{i}^{a}(P)$, to the atomic orbital momentum density $\rho_{i}(P)$ :

$$
G_{i}(P) \equiv \frac{\rho_{i}^{a}(P)}{\rho_{i}(P)} .
$$

We calculate these factors in the Hartree-Fock (HF) approximation, or (for hydrogen) more accurately, using many-body perturbation theory (MBPT). For example, Fig. 2 shows two different adjustment factors for atomic hydrogen. The dashed line shows the simplest adjustment factor, $G^{\mathrm{HF}}(P)$, that results from calculating $\rho^{a}{ }_{1 \mathrm{~s}}(P)$ using the amplitude $A_{n}(\mathbf{P})$ with the positron wavefunction $\varphi_{\mathbf{k}}$ in the static field of the atom. The solid line corresponds to the near-exact factor, $G^{\mathrm{MBPT}}(P)$, obtained in the MBPT calculation which uses a fully correlated positron wavefunction (Dyson orbital) and includes all orders of the expansion of the annihilation vertex [7,8]. These factors have the effect of suppressing the high momentum regions of the $\gamma$-spectra.

\section{Results}

The annihilation spectra for $\mathrm{H}_{2}$ are shown in Fig. 3(a). The plane-wave calculation (thin solid line) gives a spectrum with a FWHM of $2.14 \mathrm{keV}$ compared to the experimental result of $1.59 \mathrm{keV}$ (circles) [1]. For $\mathrm{H}_{2}$ the simplest $\mathrm{HF}$ adjustment factor narrows the spectrum to $1.88 \mathrm{keV}$. Inclusion of the "exact" MBPT adjustment factor in the calculation narrows the PW result significantly to $1.50 \mathrm{keV}$, providing a FWHM that is within $5 \%$ of experiment. It is clear that the MBPT is much superior in describing the spectrum for Doppler shifts below $2 \mathrm{keV}$, while in the wings the experimental data are between the two calculations.

For molecules containing atoms with larger atomic numbers, however, the effect of the nuclear repulsion accounted for by the HF factors will dominate over the additional electron-positron correlation effects included in the MBPT adjustment factor.

Fig. 3(b) shows the spectrum for $\mathrm{CF}_{4}$. The LCAO calculation (dashed line) produces a spectrum that is in reasonable agreement with the more sophisticated DFT based B3LYP/TZVP model result (thin solid line) with a FWHM of $4.29 \mathrm{keV}$. Inclusion of the adjustment factor $G^{\mathrm{HF}}(P)$ narrows the spectrum considerably to $3.17 \mathrm{keV}$, bringing it in closer agreement with the experimental value of $2.92 \mathrm{keV}$. Similar results are found for other molecules. The PW DFT based B3LYP/TZVP model calculation produces FWHM that are 30-40\% larger than in experiment (Fig. 1). Our calculations using the adjustment factors, for $\mathrm{H}_{2}, \mathrm{CF}_{4}$ and other molecules [9], show that this is mostly due to the neglect of the nuclear repulsion, with electron-positron correlations playing a relatively minor role. 


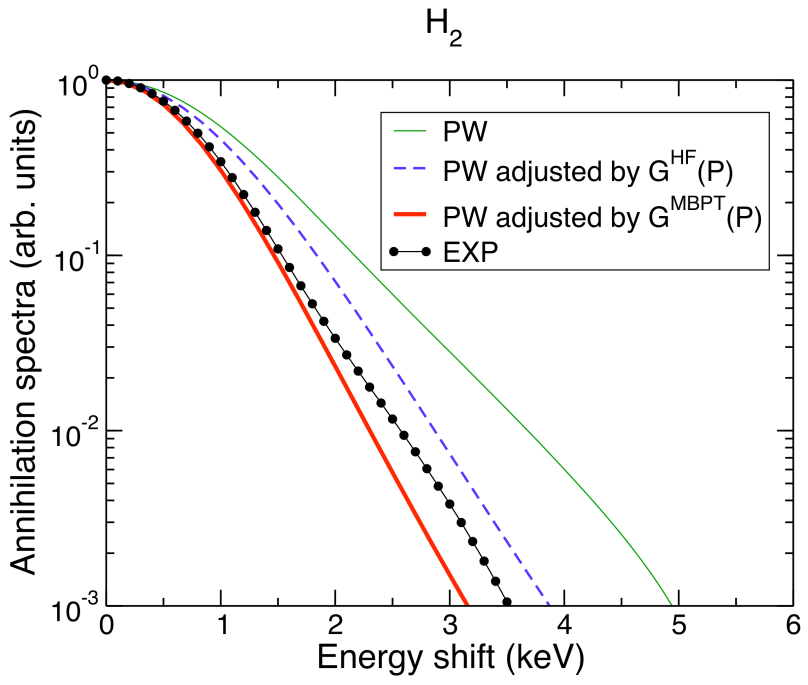

(a)

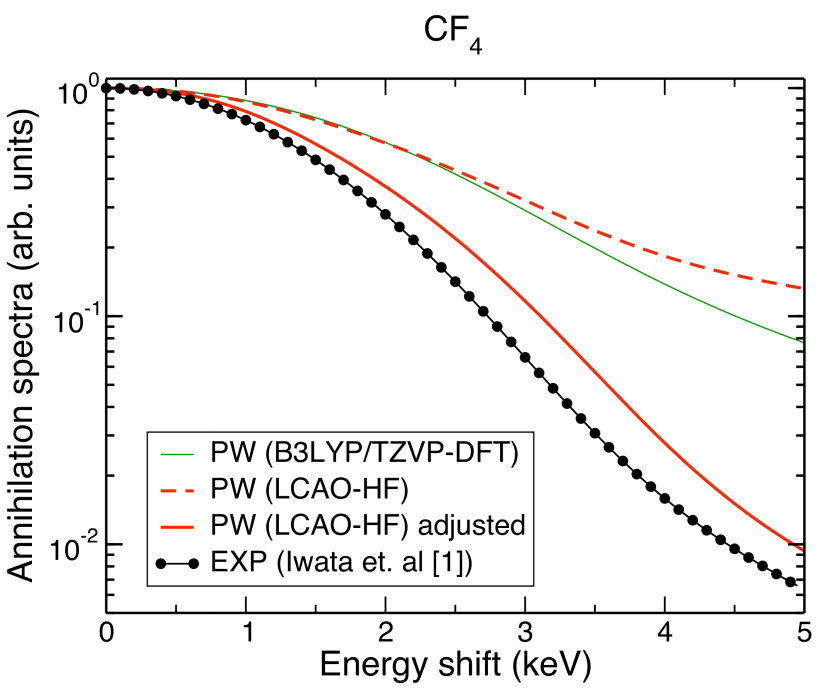

(b)

Figure 3: Annihilation $\gamma$-spectra for (a) $\mathrm{H}_{2}$ and (b) $\mathrm{CF}_{4}$ showing the plane-wave calculation (thin solid line) and the effect of the atomic adjustment factors (thick solid line). Experimental data (circles) are two-Gaussian fits of the annihilation spectra from Ref. [1].

\section{Conclusions}

We have performed calculations of $\gamma$-spectra for annihilation on a selection of molecules including methane and its fluorosubstitutes. The calculations using the electron momentum densities from the DFT B3LYP/TZVP model and unit positron wavefunction give linewidths that are approximately $30 \%$ larger than experiment. Through the adjustment factor calculations we have found that the main source of the discrepancy between the linewidths is due to the neglect of the positron repulsion from the nucleus. The repulsion suppresses the positron wavefunction at small positronnuclear separations, effectively reducing the probability of annihilation on high momentum core electrons. Furthermore, we have shown that the effective distortion of the electron momentum density, when it is observed through positron annihilation spectra, can be approximated by a relatively simple scaling factor.

Ideally, when performing a molecular calculation, one would like to incorporate a positron wavefunction that at least accounts for the electrostatic interaction. However, if this is not feasible, for example, for large systems, then one can instead incorporate the adjustment factors or at the very least, the scaling factor from Fig.1. Realistic annihilation $\gamma$-spectra linewidths can therefore be obtained from relatively simple (plane wave) independent particle model calculations. Moreover, the simple scaling factor allows for the deduction of electron momentum densities from positron annihilation $\gamma$-spectra.

\section{References}

[1] K. Iwata, R. G. Greaves and C. M. Surko: Phys. Rev. A Vol. 55 (1997), p. 3586

[2] S. Y. Chuang and B. G. Hogg: Can. J. Phys. Vol. 45 (1967), p. 3895

[3] R. Ferrell: Rev. Mod. Phys. Vol. 28 (1956), p. 308

[4] M. J. Puska and R. M. Nieminen: Rev. Mod. Phys. Vol. 66 (1994), p. 841

[5] F. Wang: J. Phys. Chem. A Vol. 107 (2003), p. 10199

[6] P. Kaijser, V. H. Smith Jnr.: Adv. Quantum Chem. Vol. 10 (1977), p. 37

[7] G. F. Gribakin and J. Ludlow: Phys. Rev. A Vol. 70 (2004), p. 032720

[8] L. J. M. Dunlop and G. F.Gribakin: J. Phys. B Vol. 39 (2006), p. 1647

[9] D. G. Green, S. Saha, F. Wang, G. F. Gribakin and C. M. Surko: (in preparation 2010) 